\title{
A study of the dose-response relationship for transscleral cyclophotocoagulation in refractory glaucoma
}

\section{Yao Xu}

First Affiliated Hospital of Soochow University https://orcid.org/0000-0002-4824-3701

Wei Shen ( $\sim$ guderian@suda.edu.cn )

https://orcid.org/0000-0002-6532-3914

\section{Research article}

Keywords: transscleral cyclophotocoagulation procedure; refractory glaucoma; dose-response relationship

Posted Date: September 11th, 2019

DOI: https://doi.org/10.21203/rs.2.14306/v1

License: (c) (1) This work is licensed under a Creative Commons Attribution 4.0 International License. Read Full License 


\section{Abstract}

Background To research the efficiency of transscleral cyclophotocoagulation (TCP) in refractory glaucoma and to evaluate the dose-response relationship.

Methods A retrospective analysis of the cases of 31 eyes of 31 consecutive patients with uncontrolled glaucoma who underwent TCP. Six to thirty-six months were reviewed. The main outcome measure is intraocular pressure (IOP) reduction combined with the best corrected visual acuity and complications. Calculations were performed to determine possible predictive factors that would help establish optimal treatment parameters.

Results All the 31 patients felt a relieved ocular pain after TCP. Corneas of 30 eyes recovered transparent one week after TCP, except one eye with corneal epithelialization. The IOP of all patients decreased to $5 \mathrm{mmHg}-38 \mathrm{mmHg} \rrbracket$ average is $17.93 \pm 8.00 \mathrm{mmHg}$ at the end of follow-up. The main earlier complications include conjunctival burns, hyphema and mild inflammation of the anterior chamber, and the late complications were phthisis bulbi and corneal staphyloma. Final IOP was moderately negative related to the number of spots, $r=-0.41(t=2.05, P<0.05)$, and the linear regression equation is $y=27.53-0.62 x$ (independent variable $x$ represents the number of spots, dependent variable y represents the final IOP). To a medium extent, the final IOP was negatively correlated with the number of the audible spots, $r=-0.58(t=3.75, P<0.01)$, and the linear regression equation is $y=28.39-1.26 x$.

Conclusion Cyclophotocoagulation is a convenient, safe and effective procedure in refractory glaucoma. The recommended parameter is about 14 spots or 8 audible spots.

\section{Background}

Refractory glaucoma refers to glaucoma which is difficult to control neither by medication nor by surgery. The causes of refractory glaucoma vary, including Pars Plana Vitrectomy (PPV), ocular traumatic operation and different kinds of anti-glaucoma surgery. As ocular enucleation is no longer a conventional therapy for refractory glaucoma nowadays, we are confronted with the demand for well controlled IOP, less ocular pain and better appearance of eyeball. TCP has been adopted worldwide, meanwhile, complications are also reported, such as visual loss, intense postoperative pain, uveitis, uncontrolled IOP, phthisis bulbi and so on. The aim of this report is to evaluate the correlation between the laser dose and the reduction of IOP as well as to identify the possible predictive factors that could achieve optimal outcome.

\section{Methods}

\section{Patient involvement}

Patients were not directly involved in the design of this study. 


\section{Data source}

The study is a retrospective case review of 31 patients (31 eyes) who underwent TCP with the semiconductor diode 810nm laser and the contact G-probe (IRIS Oculight SLx, IRIS Medical Inc., Mountain View, CA) at the First Affiliated Hospital of Soochow University, Suzhou, China, from August 2011 to July 2014. 16 males and 15 females; 16 left eyes and 15 right eyes; ages range from 35 years old to 83 years old (average was 60.81 \pm 13.78 ); diagnoses are shown in Table 1. Neovascular glaucoma in 19 eyes, 6 suffered from secondary glaucoma, 4 with mixed glaucoma and 2 were diagnosed as residual glaucoma. 16 eyes had received ocular operations before (1 to 4 times), which were involved with surgeries after trauma, keratoplasty, trabeculectomy, Ahmed glaucoma valve implantation, PPV, silicone oil tamponade and removal, cataract extraction, intraocular lens implantation, retinal photocoagulation etc.

Average IOP after the maximal medication was over $60 \mathrm{mmHg}$ in 16 eyes, $27 \mathrm{mmHg}-59 \mathrm{mmHg}$ in 8 eyes, 1 eye with opaque cornea after keratoplasty and severe corneal edema presented in 6 eyes (finger touch IOP: $T n+2-T n+3)$. No light perception in 22 eyes, light perception in 3 eyes, hand motion in 3 eyes, counting finger in 2 eyes and 1 eye with a visual acuity of 0.25 . All patients suffered from severe ocular pain, headache, conjunctival congestion. Examinations with gonioscope in some approachable cases showed widely adhesion, angle recession, massive new blood vessels, pigmentation and residual silicon oil.

TCP was performed with the semiconductor diode $810 \mathrm{~nm}$ laser and the contact G-probe with the same experienced oculist. Procedures were conducted after retrobulbar anesthesia with $2 \%$ lidocaine. The $G$ probe was placed closely to the limbus with an axial orientation to match the scleral curvature. Duration was set at $2000 \mathrm{~ms}$ and the initial power setting was $1750 \mathrm{~mW}$. The power was increased by $250 \mathrm{~mW}$ until an audible spot (caused by tissue explosion of the ciliary process, the iris root anteriorly or the retina posteriorly) was heard, then reduced the power by $250 \mathrm{~mW}$ and completed the operation at this energy level. The indeed power was about $1750 \mathrm{~mW}-3000 \mathrm{~mW}$ (average was $2341.94 \pm 311.47 \mathrm{~mW}$ ). In early cases, laser was applied over 360 degrees of the ciliary body, sparing the 3 and 9 o'clock regions (avoid injuring the long posterior ciliary vessels). The number of the spots is from 18 to 26 . However, phthisis bulbi was seen in several eyes. Then a half treatment dose was taken, that is, the number of the spots is about 812. Yet, the decreased IOP is not satisfying. We adopted two-thirds dose finally (14-17 spots) and got a reasonable IOP. The number of spots and the energy per spot were suggested slightly decreased in patients with injured ciliary body, especially those who have a better vision.

Patients received no special treatment before TCP. TobraDex was topically prescribed three times daily for 1-2 weeks, companied with atropine once daily for several days post operation. Glaucoma medications were discontinued during follow-up based on the IOP reduction achieved.

Subjective symptoms, the best corrected visual acuity (BCVA), IOP and ocular signs as well as complications were documented at the $1^{\text {st }}$ day, the $1^{\text {st }}$ week, the $1^{\text {st }}$ month, the $3^{\text {rd }}$ month and consecutive 
visit (every 3 months) post operation. All 31 patients were evaluated with a mean follow-up of $13.23 \pm 7.84$ months (range from 6 to 36 months).

Schiote's ${ }^{[1]}$ operation success standard was accepted, though we modified that the number of the eye drops added after TCP for patients with or without light perception was not limited in consideration of the aim of TCP. Success was defined as total relief of pain, as well as the IOP was less than $40 \mathrm{mmHg}$ in eyes of no light perception; or a final IOP is between 5 and $21 \mathrm{mmHg}$ in eyes with a visual acuity of better than light perception. General glaucoma medications were no longer applied in all patients after TCP, except topical glaucoma eye drops. Final IOP was obtained at the 3rd month after TCP.

\section{Statistical analysis}

We performed data analysis using SPSS Statistics 22 (SPSS Inc., Chicago, Illinois, USA). Linear regression analysis was used to evaluate the dose-response relationship between the number of spots/ the number of audible spots and the final IOP. Statistical methods included ANOVA and T test, P-value $<0.05$ was considered as significant difference.

\section{Results}

Ocular pain occurred to just one patient during the TCP. Great relief of pain could be seen in 28 patients at the $1^{\text {st }}$ day after TCP, and all 31 patients had no complaints 3 days later.

At the $1^{\text {st }}$ day after TCP, IOP in one eye with corneal epithelization after keratoplasty was Tn. Obvious corneal opacity were found in 5 eyes, corresponding intraocular pressure from $\mathrm{Tn}$ to $\mathrm{Tn}+1$. IOP of other 25 eyes fluctuated between $11 \mathrm{mmHg}$ and $59 \mathrm{mmHg}$. IOP of all eyes decreased post operation. Cornea of 30 eyes turned totally apparent at the $1^{\text {st }}$ week after TCP, and average IOP was $21.60 \pm 8.71 \mathrm{mmHg}$ (range $10 \mathrm{mmHg}$ to $58 \mathrm{mmHg}$ ); Average IOP was $22.13 \pm 6.91 \mathrm{mmHg}$ (range $8-42$ ) at the $1^{\text {st }}$ month; Average IOP was $17.93 \pm 8.00 \mathrm{mmHg}$ (range 5-38) at the end of the follow up. None IOP in these cases was higher than $40 \mathrm{mmHg}$. Final IOP in 3 eyes with a visual acuity better than light perception $₫ 21 \mathrm{mmHg}$. According to the former mentioned criteria, success rate in our study was $90.32 \%$.

Visual acuity remained the same as before in 30 eyes following TCP, and 1 eye showed improved visual acuity from no light perception to hand motion.

Early complications occurred in 17 eyes (the incidence rate is $54.84 \%$ ). They were: conjunctival burn in 1 eye (due to the damaged $\mathrm{G}$ probe). At the 1st day, slighter hyphema was seen in 3 eyes, insignificant bloody aqueous in 2 eyes, 2 eyes with mild exudative and 9 eyes with mild inflammation of the anterior chamber. After topical treatment on the patients, all complications disappeared in a week. Late complications occurred in 5 eyes (incidence rate is $16.13 \%$ ). All were observed in the eyes with full dose (incidence rate is $50.00 \%$ among eyes with full dose). There was phthisis bulbi in 4 eyes and mild corneal staphyloma in 1 eye that received keratoplasty 2 years before. 
Considering the most possible factors that may affect the final IOP (topical glaucoma eye drops were allowed), we divided the patients on the base of the number of spots and the audible spots. The parameters and outcomes are listed in Table 2 according to the spots number (group I presents half-dose, group II presents $2 / 3$ dose and group III presents full-dose). It turned out that the number of spots and the final IOP have a statistically significant difference $(P<0.05)$. Meanwhile, the parameters and outcomes are listed in Table 3 according to the audible spots number. The results also show that age may play a significant role besides the number of audible spots and the final IOP.

Linear regression was evaluated between the final IOP and the number of spots as well as the number of audible spots. Final IOP was negatively related to the number of spots to a medium extent, $r=-0.41 \nabla t=$ $2.05, P<0.05$, and the linear regression equation is $y=27.53-0.62 x$ (independent variable $x$ represents the number of spots, dependent variable $y$ represents the final IOP). Final IOP was also negatively correlated with the number of the audible spots, $r=-0.58 \otimes t=3.75, P<0.01 \mathrm{Z}$, and the linear regression equation is $\mathrm{y}=$ 28.39-1.26x. We could figure that the final IOP has a better correlation with the number of the audible spots than with the number of spots.

\section{Discussion}

Refractory glaucoma is a tough challenge in ophthalmological clinic. Particularly those patients with a better conjunctiva and limbus, on whom certain anti-glaucoma surgeries could still be done, such as trabeculectomy, Ahmed glaucoma valve implantation, scleral drainage and so on. The operations are deadly hard because of the varied etiologies and complicated general conditions. Meanwhile, the end results are usually not satisfying. Cyclocryotherapy has been advocated in the treatment of glaucoma at the beginning of $1930 \mathrm{~s}^{[2]}$ through destruction of the ciliary body to reduce the aqueous secretion but was restricted practiced because of poor prediction and severe complications, which include visual loss, ocular pain, uveitis, uncontrolled IOP and phthisis bulbi. Though anti-VEGF drugs provide a new way for neovascular glaucoma in the last few years, other therapies still need be added. In recent decades, TCP has become more popular because of its favorable tolerance and efficacy, in addition to its good penetration and selective absorption by the pigmented tissue of the ciliary body. Previous research also recommended that TCP with an individual treatment dosage should be favored vs Cyclocryotherapy. ${ }^{[3]}$

TCP is effective for patients with refractory glaucoma who have failed trabeculectomy or other tube shunt procedures, patients with extremely bad vision and uncontrolled IOP, and patients who need pain relief. It is suitable for patients whose general condition are too vulnerable to tolerate invasive surgery or who refuse more aggressive operations. TCP is as well helpful in emergent situations, such as the acute onset of neovascular glaucoma. TCP makes use of the thermal coagulation effect of laser which mainly absorbed by melanocytes and pigmented epithelia to cause necrosis of ciliary body, which has been confirmed by previous histological and morphological studies. ${ }^{[4,5]}$ Decrease of the IOP after pars plicata cyclophotocoagulation resulted from the reduction of aqueous secretion, whereas that after pars plana cyclophotocoagulation resulted from enhancement of the uveoscleral outflow through the enlarged extracellular space from the anterior chamber into the suprachoroidal space. The mechanisms of TCP to 
decrease IOP include: photocoagulation causes the necrosis of the pigmented epithelia and stroma of the ciliary body through thermal damage directly, leading to the decreased secretion of aqueous of ciliary process; photocoagulation can destroy the localized capillaries of the ciliary body, and the reduced blood supply results in the reduced aqueous secretion; the contraction of the ciliary body after photocoagulation increases the uveoscleral outflow of aqueous as well as reduces the trabecular reticulum obstruction through pulling the iris root back.

Laser of $810 \mathrm{~nm}$ wavelength exhibits less scleral absorption but considerably greater absorption by melanin. ${ }^{[6]}$ The probe footplate is curved spherically to match the scleral curvature, and the tip protrudes $0.7 \mathrm{~mm}$ beyond the contact surface, which indents the conjunctiva and sclera to enhance transmission. All these characteristics guarantee the destruction of ciliary body but sclera and other normal tissue architecture. ${ }^{[7]}$ In addition, sensory nerves in ciliary body are damaged as well during TCP. That is why we could find the dissociation of subjective symptoms from IOP, for example, patients after TCP with a remained IOP higher than $30.0 \mathrm{mmHg}$ even $50.0 \mathrm{mmHg}$ complain little.

Most scholars suggest that the treatment parameter of TCP is about 18-22 spots ( 270 degrees of the ciliary body, excluding the superonasal quadrant or in all four quadrants with sparing of the 3 and 9 o'clock regions). One spot has effect on an area of $2 \mathrm{~mm}$ due to thermal conduction, thus 18-22 spots represent a $40 \mathrm{~mm}$ area, almost involves the whole ciliary plicata. Full treatment dose was defined as the number of spots no less than 18 , half dose was 10 spots around and two thirds dose was about 15 spots. Full dose is excessive for patients who matter ocular appearance, for phthisis bulbi was seen in 4 eyes with full dose in our study (the rate is $40.00 \%$ among eyes with full dose). 3 patients with half dose underwent TCP again half a year later because of not well controlled IOP or were bored with multiple glaucoma eye drops, and the others who had a better visual acuity or who was with a moderately high IOP or who cared about the outlook chose half dose treatment initiatively. Two thirds dose was widely used in our later treatment, with which we got a satisfying outcome both subjectively and objectively (final IOP were well controlled with average one eye drop).

Table 2 shows that the final IOP of the three groups were significantly different $(F=7.89, P<0.01)$.. The much lower IOP of successful eyes was associated with a larger number of spots, not the average laser energy of per spot. Table 3 shows that the final IOP of the three groups were significantly different ( $F=$ $10.57, P<0.01)$. The much lower IOP of successful eyes was associated with a larger number of the audible spots, not the average laser energy of per spot. The laser energy of per spot and that of per audible spot have no statistical difference $(F=0.48,0.60 ; P>0.05)$. Fewer kinds of eye drops added to control IOP was associated with a larger number of spots as well as a larger number of the audible spots but have no statistical difference in the two groups $(F=1.28,3.21 ; P>0.05)$.

We noticed that age may play a remarkable role in the outcomes $(F=4.48, P<0.05)$ among groups based on the audible spot number, and the oldest average age was seen in the group with the largest number of audible spots. Hypothesis can't be concluded through a small number of cases in our study, though Schiote $^{[1]}$ had already observed the higher successful rate of TCP was related to the older age (55\%under 
50 years old, $83 \%$ over 50 years old). Final IOP had a moderately negatively relationship with the number of spots as well as the number of the audible spots, and better correlation with the number of the audible spots than with the number of spots. The effect of one audible spot on decreasing IOP is as twice as the effect of one spot, which is in accordance with Sanchez's finding. ${ }^{[8]}$

One limitation of our study is the absence of a control group to distinguish the complications of the treatment from the natural history of the underlying disease. Another is that patients in our study were in worse condition (IOP of 16 eyes $>60.0 \mathrm{mmHg}$, IOP of 7 patients could not be recorded due to either opaque cornea or highly edema cornea), thus IOP before and after TCP could not be compared precisely. Even in the absence of comparative data, this study still indicates TCP decreases the IOP extraordinary. According to the sound result defined before, the success rate of our study is $90.32 \%$, decreased to $74.19 \%$ considering the 5 cases with severe late complication, which is consistent with other studies. ${ }^{[9-12]}$ Nevertheless, success rate of patients with $2 / 3$ dose was as high as $91.67 \%$ (except one eye with a final $I O P>21.0 \mathrm{mmHg}$ ).

\section{Conclusion}

TCP is a simple, economic and effective treatment. It is a predictable procedure with fewer complications in refractory glaucoma that is proper for countries or regions with a large number of late-stage patients with glaucoma. Two thirds dose is recommended, that is about 15 spots or 8 audible spots. More accurate relationship between dose and response need to be evaluated with larger cases. Stabilization of IOP after TCP requires a long time. More attention is needed to patients with a good visual acuity, in case of overtreatment.

\section{Abbreviations}

TCP: transscleral cyclophotocoagulation; IOP: intraocular pressure; PPV: Pars Plana Vitrectomy; BCVA: best corrected visual acuity;

\section{Declarations}

\section{Ethics approval and consent to participate}

This is a retrospective analysis. Patients were not directly involved in the design of this study.

\section{Consent for publication}

Not applicable.

\section{Availability of data and material}


The datasets used and/or analyzed during the current study are available from the corresponding author on reasonable request.

\section{Competing interests}

The authors declare that they have no competing interests.

\section{Funding}

None.

\section{Authors' contributions}

YX and WS were involved in the conception, design, implementation, data collection and analysis of the pilot study. WS wrote the first draft of the trial protocol, and both authors provided critical revision of the protocol. YX wrote the first draft of the manuscript. YX and WS reviewed and edited the manuscript. Both authors approved the final manuscript.

\section{Acknowledgements}

None.

\section{References}

[1]Schlote T, Derse M, Rassmann K, Nicaeus T, Dietz K, Thiel HJ. Efficacy and safety of contact transscleral diode laser cyclophotocoagulation for advanced glaucoma. J Glaucoma. 2001. 10(4): 294301.

[2]Pastor SA, Singh K, Lee DA, et al. Cyclophotocoagulation: a report by the American Academy of Ophthalmology. Ophthalmology. 2001. 108(11): 2130-8.

[3]Tzamalis A, Pham DT, Wirbelauer C. Diode laser cyclophotocoagulation versus cyclocryotherapy in the treatment of refractory glaucoma. Eur J Ophthalmol. 2011. 21(5): 589-96.

[4]Liu GJ, Mizukawa A, Okisaka S. Mechanism of intraocular pressure decrease after contact transscleral continuous-wave Nd:YAG laser cyclophotocoagulation. Ophthalmic Res. 1994. 26(2): 65-79.

[5]Jian G, Mei W, Jin JC, Ping Z. Observation of short term effect of transscleral diode laser cyclophotocoagulation in patients with refractory glaucoma. Chinese Journal of Practical Opht. 1999. 
[6]Pantcheva MB, Kahook MY, Schuman JS, Noecker RJ. Comparison of acute structural and histopathological changes in human autopsy eyes after endoscopic cyclophotocoagulation and transscleral cyclophotocoagulation. Br J Ophthalmol. 2007. 91(2): 248-52.

[7]Nguyen AT, Maslin J, Noecker RJ. Early results of micropulse transscleral cyclophotocoagulation for the treatment of glaucoma. Eur J Ophthalmol. 2019: 1120672119839303.

[8]Sanchez FG, Peirano-Bonomi JC, Grippo TM. Micropulse Transscleral Cyclophotocoagulation: A Hypothesis for the Ideal Parameters. Med Hypothesis Discov Innov Ophthalmol. 2018. 7(3): 94-100.

[9]Aquino MC, Barton K, Tan AM, et al. Micropulse versus continuous wave transscleral diode cyclophotocoagulation in refractory glaucoma: a randomized exploratory study. Clin Exp Ophthalmol. 2015. 43(1): 40-6.

[10]Kuchar S, Moster MR, Reamer CB, Waisbourd M. Treatment outcomes of micropulse transscleral cyclophotocoagulation in advanced glaucoma. Lasers Med Sci. 2016. 31(2): 393-6.

[11]Tan AM, Chockalingam M, Aquino MC, Lim ZI, See JL, Chew PT. Micropulse transscleral diode laser cyclophotocoagulation in the treatment of refractory glaucoma. Clin Exp Ophthalmol. 2010. 38(3): 26672.

[12]Yelenskiy A, Gillette TB, Arosemena A, et al. Patient Outcomes Following Micropulse Transscleral Cyclophotocoagulation: Intermediate-term Results. J Glaucoma. 2018. 27(10): 920-925.

\section{Tables}

Table 1. Diagnoses of the Included Patients

\begin{tabular}{|l|l|}
\hline Kinds of Glaucoma & Number \\
\hline Neovascular glaucoma & 19 \\
\hline Secondary glaucoma & 6 \\
\hline mixed glaucoma & 4 \\
\hline residual glaucoma & 2 \\
\hline
\end{tabular}

Table 2. Groups based on the spots number 


\begin{tabular}{|l|l|l|l|l|l|l|}
\hline & Case number & The spots number & Age & Laser energy & Final eye drops & Final IOP \\
\hline I & 8 & $\begin{array}{l}9.25 \pm 2.05 \\
(5 \square 12)\end{array}$ & $53.63 \pm 8.38$ & $2281.25 \pm 196.28$ & $1.25 \pm 1.16$ & $24.50 \pm 9.07$ \\
\hline II & 12 & $\begin{array}{l}14.92 \pm 1.00 \\
(14 \square 17)\end{array}$ & $59.33 \pm 18.09$ & $2370.83 \pm 340.76$ & $0.92 \pm 0.90$ & $17.33 \pm 3.37$ \\
\hline & & $21.50 \pm 3.37$ & $68.60 \pm 7.83$ & $2415.00 \pm 319.77$ & $0.50 \pm 1.08$ & $13.40 \pm 7.96$ \\
\hline III & 10 & $(19 \square 29)$ & & & & \\
\hline
\end{tabular}

Table 3. Groups based on the audible spots number

\begin{tabular}{|l|l|l|l|l|l|l|}
\hline & Case number & The audible cues number & Age & Laser energy & Final eye drops & Final IOP \\
\hline I & 8 & $\begin{array}{l}3.88 \pm 1.13 \\
(2 \square 5)\end{array}$ & $61.00 \pm 11.03$ & $2306.25 \pm 314.46$ & $1.00 \pm 1.20$ & $23.38 \pm 7.52$ \\
\hline II & 16 & $\begin{array}{l}8.44 \pm 1.26 \\
(6 \square 10)\end{array}$ & $55.94 \pm 14.71$ & $2415.63 \pm 297.05$ & $1.13 \pm 1.02$ & $18.75 \pm 6.04$ \\
\hline III & 6 & $\begin{array}{l}13.67 \pm 2.25 \\
(11 \square 16)\end{array}$ & $74.00 \pm 5.83$ & $2291.67 \pm 293.97$ & $0.00 \pm 0.00$ & $8.50 \pm 5.09$ \\
\hline F & & & & & \\
\hline$P$ & & $<2.22$ & 4.84 & 0.60 & 3.21 & 10.57 \\
\hline
\end{tabular}

